\title{
25 Jahre nach dem Mauerfall: Zum Stand der Aufarbeitung der Psychiatrie in der DDR
}

\author{
25 Years After the Wall Came Down: Psychiatry in East Germany Revisited
}

Autoren

Institute

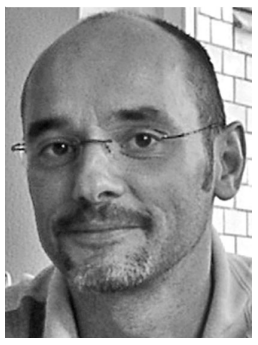

Holger Steinberg

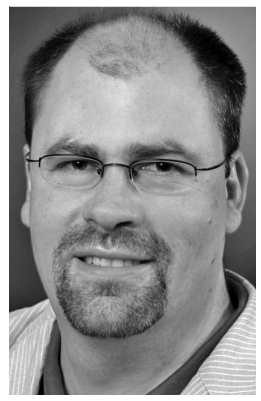

Ekkehardt Kumbier

Bibliografie

Dol http://dx.doi.org/

10.1055/s-0034-1387554

Psychiat Prax 2015; 42:

119-121

(c) Georg Thieme Verlag KG

Stuttgart · New York

ISSN 0303-4259

Korrespondenzadresse PD Dr. Holger Steinberg

Archiv für Leipziger

Psychiatriegeschichte

Klinik und Poliklinik für

Psychiatrie und Psychotherapie

Medizinische Fakultät der

Universität Leipzig

Semmelweisstraße 10

04103 Leipzig

holger.steinberg@medizin.uni-

leipzig.de

Holger Steinberg ${ }^{1}$, Ekkehardt Kumbier ${ }^{2}$

Archiv für Leipziger Psychiatriegeschichte, Klinik und Poliklinik für Psychiatrie und Psychotherapie, Medizinische Fakultät der Universität Leipzig

AG Philosophie und Geschichte der Psychiatrie, Klinik und Poliklinik für Psychiatrie und Psychotherapie, Universitätsmedizin Rostock

Vor einem Vierteljahrhundert fiel die Berliner Mauer und die DDR hörte auf zu existieren. Dieses Ereignis war in den Medien in den letzten Monaten sehr präsent. Die Fragen, ob die DDR ein Unrechtsstaat war oder was es signalisiert, wenn ein Linker Ministerpräsident eines Bundeslandes wird, bewegen die Nation.

Ein solcher Jahrestag legt auch für unser Fach die Frage nahe, wie der Stand der historischen Aufarbeitung der Psychiatrie in der DDR ist. Hat die Forschung Vorurteile revidiert oder bestätigt, hat sie unser Wissen erweitert oder verändert?

Einer der drängendsten Aspekte war die Frage, ob es einen systematischen staatlichen Missbrauch der Psychiatrie gegeben hatte. Süß hatte 1998 nach Durchsicht hunderter Akten des Ministeriums für Staatssicherheit (MfS) die Diskussion eröffnet [1]. Zwar waren Ärzte als inoffizielle Mitarbeiter des MfS tätig und hatten Patientengeheimnisse verraten, waren psychisch Kranke vorübergehend gegen ihren Willen untergebracht worden, und es hatte Fälle psychiatrischer Disziplinierung unbequemer Menschen gegeben. Dennoch stellte Süß klar, dass die Institution Psychiatrie nicht systematisch als Instrument zur Verfolgung politischer Gegner benutzt wurde. Mit dieser Aussage sind nicht alle einverstanden, sodass Süß z.T. heftig kritisiert wurde; nach heutigem Kenntnisstand zu Unrecht, denn es gibt bisher keine quellenfundierte Studie, die das Gegenteil beweist.

Immerhin folgte ihrer Publikation eine Anzahl von Monografien, die auf den Komplex Ärzteschaft und MfS fokussierten. Weil gelangte hinsichtlich der Spitzeltätigkeiten zu ähnlichen Einschätzungen wie Süß. Die Mehrheit der Ärzte habe sich darauf nicht eingelassen, wenngleich der Anteil an inoffiziellen Mitarbeitern des MfS unter Ärzten höher als in der Gesamtbevölkerung war. Die Leipziger Historikerin sieht die Ursache darin, dass die Partei- und Staatsführung diese bildungsbürgerlich und ständisch geprägte Be- rufsgruppe besonders observieren ließ. Sie hat auch festgestellt, dass die Psychiater im Vergleich zu anderen Ärzten besonders häufig vom MfS beobachtet wurden. Laut Weil standen Psychiater stärker als andere Mediziner im Fokus der Staatssicherheit, weil sie auch gerichtlich untergebrachte bzw. „politisch relevante“ Patienten beobachten sollten [2].

Die „Operative Psychologie“ und die wissenschaftlichen Ergebnisse des gleichnamigen Lehrstuhls des MfS hat Richter untersucht [3]. Behnke und Fuchs legten eine Studie vor, die zeigt, wie das MfS mithilfe „der psychologischen Kriegsführung im Innern“ agierte. Diese habe die Zwangseinweisung von Andersdenkenden in psychiatrische Kliniken inbegriffen [4]. Beide Bücher vermitteln den Eindruck, dass die Stasi Psychologie und Psychiatrie instrumentalisierte und als Kampfmittel betrachtete, sie auf empirisch-wissenschaftlicher Basis für ihre Zwecke dienstbar machen wollte.

Zur Psychiatrie in der DDR gehört als bis z.T. heute wirkendes Erbe das psychische Leid unter der Diktatur. Priebe et al. geben sachkundig Einblick in die Spezifik der Therapie und Begutachtung in der DDR politisch Verfolgter, und auch sie wenden sich besonders der Rolle von MfS-Psychiatern zu [5].

Patientengeschichten finden sich im Sammelband von Müller und Mitzscherlich [6]. Sie lassen zudem Zeitzeugen aus der Pflege- und Ärzteschaft über ihre Erfahrungen berichten. Mögen diese nun stets DDR-typisch sein oder nicht, auf jeden Fall stellen diese subjektiven Sichten Quellen eigener und wichtiger Art dar, die lange in der Psychiatriegeschichtsforschung vernachlässigt wurden. Das Buch ermöglicht einen unverstellten Blick in psychiatrische Kliniken, die Versorgungsnöte des Alltags, in Behandlungserfahrungen. Überdies liegen die Perspektiven einer Schwester und eines Psychiaters als autobiografische Quellen vor $[7,8]$. 
Als eines der noch wenigen, aber gelungenen Beispiele für Institutionsgeschichte in der DDR sei Roses Buch über die brandenburgischen Kliniken gewürdigt [9]. Der Potsdamer Historiker macht quellengestützt deutlich, vor welchen Problemen die brandenburgischen Versorgungskliniken standen. Rose legt die Vernachlässigung der Psychiatrie und erst recht der „Anstalten“ durch die offizielle Gesundheitspolitik offen. Zunehmende Mangelwirtschaft und Stigmatisierung psychisch Kranker sind nur zwei Ursachen dieser destruktiven Entwicklung. Der Eindruck aus diesem Buch dürfte sich auf die Versorgungslandschaft der DDR erweitern lassen. Auch Hanrath geht auf dieses Thema ein [10]. Sie hat die Anstaltsfürsorge in der DDR und BRD anhand von zwei psychiatrischen Kliniken während der ersten beiden Nachkriegsjahrzehnte verglichen. Sie beschreibt Gemeinsamkeiten und Unterschiede, stellt grundsätzlich aber fest, dass sich die Psychiatrie in beiden Staaten nicht voneinander getrennt entwickelt habe, vielmehr aufgrund gemeinsamer Wurzeln und Kontinuitäten miteinander verbunden geblieben sei.

Das Wissen über die unmittelbare Nachkriegsgeschichte psychiatrischer Krankenhäuser in den verschiedenen Besatzungszonen ist bisher aber gering. Neugierig macht deshalb die derzeit laufende, von der DFG geförderte Untersuchung von Rotzoll und Mitarbeitern. Sie untersuchen u. a. für die SBZ (Sowjetische Besatzungszone) die Überwindung der Folgen des Nationalsozialismus am Beispiel einer ehemaligen Tötungsanstalt sowie die Neuorientierung der Anstaltspsychiatrie.

Wenig wurde auch zu Therapien oder bestimmten Therapieformen gearbeitet - hier besonders somatischen, die mit der Psychiatrie in der DDR assoziiert werden. Ein Anfang wurde mit der Pawlow'schen Schlaftherapie gemacht [11]. Rzesnitzek beschäftigt sich derzeit mit der Frage, in welchem Umfang bis in die frühen 1960er-Jahre hinein Schocktherapien (Insulin-Koma-Therapie, Cardiazol- und Elektrokrampftherapie) und die Leukotomie oder stereotaktische Methoden angewendet wurden. Über die Herstellung und Verfügbarkeit der Psychopharmaka in der DDR haben Hess und Mitarbeiter publiziert [12-15]. Damit schlossen sie Wissenslücken und revidierten manches Vorurteil etwa hinsichtlich einer vollständigen Rückschrittlichkeit der ostdeutschen Pharmakopsychiatrie. Die Arbeitsgruppe des Instituts für Geschichte der Medizin und Ethik in der Medizin der Charité hatte erfolgreich ein DFG-Projekt eingeworben. Ihr Vorhaben, konkret am Beispiel der Antidepressiva und Anxiolytika die klinische Einführung anhand von Patientenakten nachzuvollziehen, stieß angesichts der Leonhard'schen Klinik und Klassifikation auf Schwierigkeiten. Zur Psychotherapie liegt Geyers opulentes Sammelwerk vor [16]. Der von Zeitzeugen erarbeitete Band stellt viele Ansätze an unterschiedlichen Orten dar. Indes ist die geäußerte Kritik nachvollziehbar. Die meisten Beiträge orientieren sich nicht an geschichtswissenschaftlichen Standards, sie tragen vielmehr den Charakter persönlicher Betrachtungen. In diesem Sammelband sowie in Bernhardts und Lockots analysehistorischem „Mit ohne Freud“ [17] wird aber klar, vor welchen ideologischen Schwierigkeiten und Beschränkungen psychotherapeutische Schulen, die Psychosomatik und die Psychoanalyse standen. Beide Bücher stellen evidente Beiträge zur Aufarbeitung des parteilich-staatlichen Einflusses auf die ärztlichen Wissenschaften und das ärztliche Handeln in der DDR dar. In diesen Themenbereich fallen auch Forschungen zur politisch motivierten Personalpolitik, Fächerdifferenzierung der Psycho- und Neurofächer oder Publikationslimitierung sowie zur Rolle der Fachgesellschaft für Neurologie und Psychiatrie der DDR [18-23].
Die sozialpsychiatrischen Reformbemühungen in der DDR, v.a. die Rodewischer Thesen sind u.a. von Schmiedebach et al. und Hennings untersucht worden [24, 25]. Armbruster befasste sich mit der Arbeitstherapie, die im Spannungsfeld von Rehabilitation und Missbrauch stand [26].

Die Bearbeitung des schließlich staatlich tabuisierten Themas Suizidalität hat mit Grashoffs Monografie eine wegweisende Grundlage erhalten [27]. Er geht der Frage nach, warum die DDR eine der höchsten Suizidraten aufwies und stützt sich dabei auf die Auswertung mehrerer tausend Fälle. Demnach scheint eine Erklärung des Phänomens und ein direktes Bindeglied zwischen Unfreiheit, Pression und Suizidalität nicht offen auf der Hand zu liegen. Enger an psychiatrisch-fachwissenschaftliche Diskurse schließt sich die Chronologie zur DDR-Depressionsforschung von Thormann et al. an das Thema Depression und Suizidalität an [28]. Auch diese Arbeit zeigt, dass die Psychiatrie in der DDR Spezifika aufwies, die aber trotz Beschränkungen niemals ein dermaßen isoliertes Dasein führte, dass man von einer in sich abgeschlossenen und nur aus sich allein heraus erklärbaren DDRPsychiatrie sprechen kann, die losgelöst von der restlichen Welt existierte. Im Gegenteil entstanden auch unter schwierigen Bedingungen Beiträge ostdeutscher Psychiater, die von Weitsicht und Evidenz zeugen. Sie fanden zunächst nur in Ausnahmefällen international Beachtung. Mit historischem Abstand werden diese Arbeiten wiederentdeckt: Karl Leonhards Klassifikation der endogenen Psychosen mag das eindrücklichste Beispiel sein, Rudolf Lemkes Konzept der „Vegetativen Depression“ oder Helmut Rennerts Universalgenese der endogenen Psychosen mögen als solche erst jüngst herausgearbeitete innovative Beiträge gelten $[29,30]$; wohl auch, wenngleich nicht frei vom Einfluss des Systems, Christa Kohlers „Kommunikative Psychotherapie“ [31, 32]. Der Überblick zeigt, dass mittlerweile einige grundlegende und richtungsweisende Arbeiten vorliegen. Manches Material zur Überprüfung von Vorurteilen liegt vor, auf einigen Gebieten konnte das Wissen vertieft werden. Es wird zugleich deutlich, dass noch weiße Flecke bestehen und eine systematische Aufarbeitung bisher fehlt. Zu einem Teil mag dieses etwas ernüchternde Resümee seine Ursache darin haben, dass viele Autoren nur an einzelnen Projekten arbeiteten, so etwa zur Promotion, und sich die Forschung zur DDR nur an drei Zentren zu verstetigen scheint: der genannten Berliner Gruppe, der AG Philosophie und Geschichte der Psychiatrie an der Psychiatrischen Universitätsklinik Rostock sowie am Archiv für Leipziger Psychiatriegeschichte an der Psychiatrischen Universitätsklinik Leipzig. Die beiden Letzteren arbeiten derzeit u.a. zur sozialpsychiatrischen Reformbewegung der 1960er-Jahre [33, 34].

Die Aufarbeitung der Geschichte der Psychiatrie in der DDR wird erfreulicherweise immer wieder von deutschsprachigen Fachzeitschriften aufgegriffen. Das regt die Forschung und Diskussion an. Dafür sei eben hier den Herausgebern der „Psychiatrischen Praxis“ ausdrücklich gedankt.

\section{Literatur}

1 Süß S. Politisch mißbraucht? Psychiatrie und Staatssicherheit in der DDR Berlin: Ch. Links; 1998

2 Weil F. Zielgruppe Ärzteschaft. Ärzte als inoffizielle Mitarbeiter des Ministeriums für Staatssicherheit. Göttingen: V\&R unipress; 2008

3 Richter H. Die operative Psychologie des Ministeriums für Staatssicherheit der DDR. Frankfurt am Main: Mabuse; 2001

4 Behnke K, Fuchs J Hrsg. Zersetzung der Seele: Psychologie und Psychiatrie im Dienste der Stasi. Hamburg: Rotbuch; 1995

5 Priebe S, Denis $D$, Bauer $M$, Hrsg. Eingesperrt und nie mehr frei: psychisches Leiden nach politischer Haft in der DDR. Darmstadt: Steinkopff; 1996 
6 Müller TR, Mitzscherlich B. Psychiatrie in der DDR: Erzählungen von Zeitzeugen. Frankfurt am Main: Mabuse; 2006

7 Groß FR. Jenseits des Limes: 40 Jahre Psychiater in der DDR. Bonn: Psychiatrie; 1996

8 Zorn U. Tagebuch einer Krankenschwester: Eine Zeitreise durch die Psychiatrie. Remscheid: Re Di Roma; 2010

9 Rose W. Anstaltspsychiatrie in der DDR: die brandenburgischen Kliniken zwischen 1945 und 1990. Berlin: Be.bra-Wiss; 2005

10 Hanrath S. Zwischen „Euthanasie“ und Psychiatriereform: Anstaltspsychiatrie in Westfalen und Brandenburg: Ein deutsch-deutscher Vergleich (1945-1964). Paderborn: Schöningh; 2002

11 Scholtz D, Steinberg $H$. Die Theorie und Praxis der Pawlow'schen Schlaftherapie in der DDR. Psychiat Prax 2011; 38: 323 - 328

12 Hess V. Psychochemicals crossing the wall. Die Einführung der Psychopharmaka in der DDR aus der Perspektive der neueren Arzneimittelgeschichte. Medizinhist J 2007; 42: 61 -84

13 Klöppel U, Balz V. Psychopharmaka im Sozialismus. Arzneimittelregulierung in der Deutschen Demokratischen Republik in den 1960er Jahren. Ber Wiss 2010; 33: $382-400$

14 Balz V, Hoheisel M. East-Side story: the standardisation of psychotropic drugs at the Charite Psychiatric Clinic, 1955-1970. Stud Hist Philos Biol Biomed Sci 2011; 42: 453-466

15 Klöppel U. 1954 - Brigade Propaphenin arbeitet an der Ablösung des Megaphen. Der prekäre Beginn der Psychopharmakaproduktion in der DDR. In: Eschenbruch N, Balz V, Klöppel U et al., Hrsg. Arzneimittel des 20 Jahrhunderts: historische Skizzen von Lebertran bis Contergan. Bielefeld: transcript; 2009: 199-227

16 Geyer M, Hrsg. Psychotherapie in Ostdeutschland: Geschichte und Geschichten 1945-1995. Göttingen: Vandenhoeck \& Ruprecht; 2011

17 Bernhardt H, Lockot R, Hrsg. Mit ohne Freud - Zur Geschichte der Psychoanalyse in Ostdeutschland. Gießen: Psychosozial; 2000

18 Steinberg H, Weber MM. Vermischung von Politik und Wissenschaft in der DDR. Die Untersuchung der Todesfälle an der Leipziger Neurologisch-Psychiatrischen Universitätsklinik unter Müller-Hegemann 1963. Fortschr Neurol Psychiatr 2011; 79: 561 - 569

19 Scholz A, Wunderlich P. Möglichkeiten und Grenzen des Publizierens in der DDR. Dtsch Med Wochenschr 2000; 125: 1131-1132

20 Teitge M, Kumbier E. Zur Geschichte der DDR-Fachzeitschrift Psychiatrie, Neurologie und medizinische Psychologie (1949-1990). Nervenarzt; Doi: 10.1007/s00115-014-4198-0

21 Kumbier E, Haack K, Zettl UK. Fächerdifferenzierung unter sozialistischen Bedingungen - Die Etablierung der Neurologie an der Universität Rostock. Fortschr Neurol Psychiatr 2009; 77 (Suppl. 01): S3 -S6
22 Kumbier E, Haack K, Herpertz SC. Franz Günther von Stockert im Spannungsfeld von Politik und Wissenschaft - Ein Beitrag zur Geschichte der Nervenheilkunde in der DDR. Fortschr Neurol Psychiatr 2009; 77: $285-288$

23 Kumbier E, Haack K. Hochschullehrer in der SBZ und DDR bis 1961: Der akademische Generationswechsel an den Universitätsnervenkliniken. Nervenarzt; Doi: 10.1007/s00115-014-4218-0

24 Schmiedebach H-P, Beddies T, Schulz J et al. Offene Fürsorge - Rodewischer Thesen - Psychiatrie-Enquete: Drei Reformansätze im Vergleich. Psychiat Prax 2000; 27: 138-143

25 Hennings L. Die Rodewischer Thesen und das Symposium über psychiatrische Rehabilitation 1963 in Rodewisch. Schriftenreihe der Deutschen Gesellschaft für Geschichte der Nervenheilkunde 2014; 20 : 385-409

26 Armbruster J, Jarisch A. Im Spannungsfeld von individueller Rehabilitation und Missbrauch: Arbeitstherapie in der DDR-Psychiatrie am Beispiel des Bezirkskrankenhauses Stralsund. Schriftenreihe der Deutschen Gesellschaft für Geschichte der Nervenheilkunde 2014; 20: $353-384$

27 Grashoff $U$. „In einem Anfall von Depression...“ Selbsttötungen in der DDR. Berlin: Ch. Links; 2006

28 Thormann J, Himmerich H, Steinberg H. Depressionsforschung in der DDR - historische Entwicklungslinien und Therapieansätze. Fortschr Neurol Psychiatr 2014; 82: 68-77

29 Kumbier E, Herpertz SC. Helmut Rennert's universal genesis of endogenous psychoses: the historical concept and its significance for today's discussion on unitary psychosis. Psychopathol 2010; 43: 335-344

30 Thormann J, Himmerich H, Steinberg H. Das Konzept der „vegetativen Depression“ (1949) von Rudolf Lemke - Ein obsoletes Konzept? Psychiat Prax 2011; 38: 91 - 96

31 Steinmetz $M$, Himmerich $H$, Steinberg $H$. Eine frühe sozialpsychiatrische Schrift in der DDR. Psychiat Prax 2013; 40: 65-71

32 Steinmetz $M$, Himmerich $H$, Steinberg $H$. Christa Kohlers „Kommunikative Psychotherapie“ - ein integratives Psychotherapiekonzept im biografischen, wissenschaftlichen und historischen Kontext. Fortschr Neurol Psychiatr 2012; 80: 250-259

33 Steinberg H. Karl Leonhard hat „kein Interesse!“ - Hintergründe über das Rodewischer Symposium aus neu aufgetauchten Quellen. Psychiat Prax 2014; 41: $71-75$

34 Kumbier E, Haack K, Steinberg H. 50 Jahre Rodewischer Thesen - Zu den Anfängen sozialpsychiatrischer Reformen in der DDR. Psychiat Prax 2013; 40: 313-320 